\title{
c-Jun phosphorylation by the human vaccinia-related kinase 1 (VRK1) and its cooperation with the N-terminal kinase of c-Jun (JNK)
}

\author{
Ana Sevilla ${ }^{1}$, Claudio R Santos ${ }^{1}$, Ramiro Barcia ${ }^{1}$, Francisco M Vega ${ }^{1}$ and Pedro A Lazo*,1 \\ ${ }^{1}$ Instituto de Biología Molecular y Celular del Cáncer, Consejo Superior de Investigaciones Cientificas (CSIC)-Universidad de \\ Salamanca, Campus Miguel de Unamuno, E-37007 Salamanca, Spain
}

\begin{abstract}
The VRK1 kinase is a novel Ser-Thr kinase in the human kinome that diverged from the casein kinase 1 branch. These kinases phosphorylate transcription factors related to stress responses, such as $\mathbf{p 5 3}$. In this report we have studied the phosphorylation of the transcription factor c-Jun in its N-terminal region. The VRK1 protein phosphorylates c-Jun with a $\mathrm{Km}$ of $0.4 \mu \mathrm{M}$, and is not inhibited by SP600125. VRK1 phosphorylates c-Jun in Ser63 and Ser73 in vitro, the same residues targeted by the N-terminal kinase of c-Jun (JNK). This phosphorylation induces the stabilization and accumulation of the cJun protein. VRK1 phosphorylates the endogenous c-Jun in Ser63. VRK1 activates c-Jun dependent transcription, which is dependent on phosphorylation of Ser63 and Ser73. The c-Jun with Ser63Ala and Ser73Ala substitutions is not transcriptionally active when cotransfected with VRK1. VRK1 interacts with c-Jun but not with JNK. The cotransfection of VRK1 and JNK has an additive effect on the transcriptional activation of c-Jun indicating that they can cooperate when both are at suboptimal dose; otherwise, maximum effect by one of them prevents the effect of the other. The VRK1-c-Jun connection represents a component of a new signaling pathway whose upstream elements remain to be identified.
\end{abstract}

Oncogene (2004) 23, 8950-8958. doi:10.1038/sj.onc.1208015 Published online 20 September 2004

Keywords: VRK1; c-Jun; JNK; transcription

\section{Introduction}

A new family of Ser-Thr kinases has been identified by the homology of their catalytic domain to the vaccinia virus B1R kinase (Nezu et al., 1997). Based on the latest information from the human kinome (Manning et al., 2002), there are three members in this family, known as vaccinia-related kinases (VRK). These proteins have a kinase domain with some distant homology to human casein kinase 1 (Lopez-Borges and Lazo, 2000; Nichols

*Correspondence: PA Lazo, IBMCC-Centro de Investigación del Cáncer, CSIC-Universidad de Salamanca, Campus Miguel de Unamuno, E-37007 Salamanca, Spain; E-mail: plazozbi@usal.es

Received 11 February 2004; revised 24 May 2004; accepted 6 July 2004; published online 20 September 2004 and Traktman, 2004) from which they diverged early in evolution (Manning et al., 2002). However, these three kinases differ significantly in their regulatory domains, which are located at the C-terminus in the case of VRK1 and VRK2, and at the N-terminus in VRK3. VRK1 is highly expressed in tumor cells with high proliferation rates (Nezu et al., 1997). In developing murine embryos, VRK1 and VRK2 are also highly expressed in midgestation, when the proliferation of hematopoietic cells is extremely high (Vega et al., 2003). Also, the substrate characteristics are different from those of casein kinase 1 (Lopez-Borges and Lazo, 2000). VRK1 is a nuclear kinase, detected in both murine and human cells, that regulates p53 by phosphorylation in Thr 18 (Lopez-Borges and Lazo, 2000; Barcia et al., 2002). Owing to the relation of $\mathrm{p} 53$ to cellular stress responses we decided to determine whether the VRK1 kinase was also able to phosphorylate other proteins, which are known to be involved in response to different types of stress signals. One of these potential candidates is the cJun transcription factor.

Many cellular responses to stress are regulated by AP1 binding sites on DNA (Eferl and Wagner, 2003). These sites are recognized by transcription complexes form by dimers of different protein families. The AP1 transcription complex is formed by a protein dimer in which there are many different subunits that can be combined (Kerppola and Curran, 1993; van Dam and Castellazzi, 2001; Dlakic et al., 2001). All of them have a DNA binding domain and a dimerization domain containing a leucine zipper. These proteins belong to any of the families of transcription factors such as JUN, FOS, ATF and MAF (musculoaponeurotic fibrosarcoma) (Eferl and Wagner, 2003). The individual members of the dimer to form a complex can belong to any of the groups; therefore, there are a very large number of combinations that might modulate gene expression. Their different combinations offer the possibility of fine tuning the control of transcription (van Dam and Castellazzi, 2001; Shaulian and Karin, 2001, 2002). The nature of the complex influences the specificity of the recognition sites; thus, the c-Jun and ATF2 heterodimer recognizes the CRE sites (cAMP response elements) (Shuman et al., 1997; Hai and Hartman, 2001). ATF2 is also phosphorylated by VRK1 in novel residues and has an additive effect with JNK (Sevilla et al., 2004). These transcription complexes are 
implicated in the control of cell proliferation, differentiation and apoptosis by regulating gene expression (Shaulian and Karin, 2001). The c-Jun protein is regulated in response to many types of signals including growth factors (Hall et al., 2003), proinflammatory cytokines (Dong et al., 2000; Weiss et al., 2000), oxidative stress (Clerk and Sugden, 1997) and UV irradiation (Adler et al., 1995).

The c-Jun protein has an amino terminal regulatory domain that is modified by phosphorylation, and a carboxy terminal DNA binding domain. The phosphorylation implies the binding of the JNK protein to its target c-Jun, and the residues phosphorylated are Ser63 and Ser73. The phosphorylated c-Jun protein is then transcriptionally active. In the cell, phosphorylation of c-Jun occurs as a response to many different types of stress signals mediated by a group of serine-threonine kinases, the mitogen-activated protein kinases (MAPK) that include ERK, and JNK (Leppa and Bohmann, 1999; Davis, 2000; Chang and Karin, 2001).

The implication of c-Jun in so many different types of cellular functions in response to a very heterogeneous group of signals suggests that it is likely that in the cell there might be more kinases implicated in regulating its transcriptional activity. Therefore, we have studied if the recently identified vaccinia-related kinase 1 (VRK1) that phosphorylates p53 (Lopez-Borges and Lazo, 2000), another molecule implicated in stress responses, could also phosphorylate the c-Jun protein. In this report it is demonstrated that VRK1 not only targets c-Jun but that it can also cooperate with the N-terminal Jun kinase.

\section{Results}

\section{VRK1 and c-Jun co-localize in the nucleus}

For a protein to be an immediate target of another means that both have to be located in the same subcellular compartment. Therefore, the subcellular location of both VRK1 and c-Jun was determined in HeLa cells. For these experiments the cells were transfected with tagged proteins and expressed from expression vectors. VRK1-myc and HA-c-Jun were transfected and their location was determined by immunofluorescence and confocal microscopy. As shown in Figure 1, both VRK1 and c-Jun are located in the nuclei, but outside the nucleolus. These data indicate that when there are high levels of both proteins they are found in the same nuclear compartment.

\section{VRK1 phosphorylates $c$-Jun in vitro and is insensitive to SP600125}

The c-Jun transcription factor is regulated by reversible phosphorylation that is carried out by different kinases depending on the type of signal. The c-Jun protein is a potential target for the nuclear VRK1 activity. Therefore, to determine if c-Jun could be phosphorylated in vitro, a kinase assay with the GST-VRK1 (full length) protein was carried out to detect the phosphorylation of
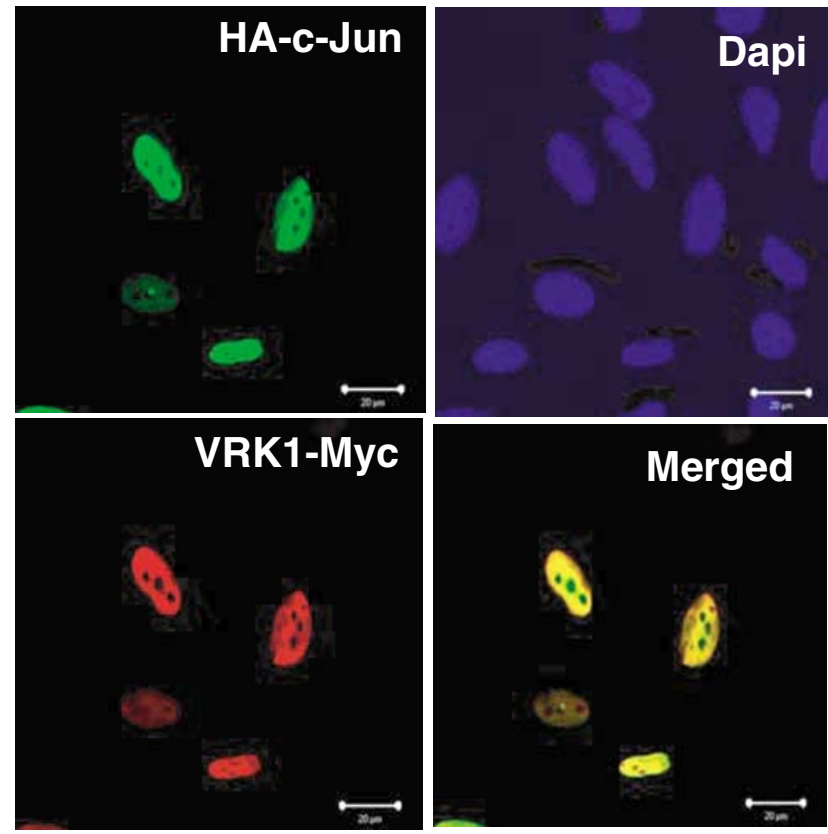

Figure 1 Nuclear colocalization of human VRK1 and c-Jun in HeLa cells. HeLa cells were transfected with VRK1 tagged with a myc epitope and c-Jun tagged with an HA epitope. Each specific epitope was detected with a primary antibody for tag followed by a different secondary antibody labeled with $\mathrm{Cy} 2$ and $\mathrm{Cy} 3$, respectively. Interphase nuclei were identified by DAPI staining

the N-terminal domain of c-Jun, as a GST-c-Jun (1-223) fusion protein. As shown in Figure 2a, VRK1 phosphorylates c-Jun but not the GST protein moiety. If the GST-VRK1 fusion protein contained a shorter segment of c-Jun (residues 1-79), phosphorylation was lost probably because the target residues are very proximal to the C-terminus of the molecule and do not fold properly (Lopez-Borges and Lazo, 2000). Next, we performed a kinase analysis where the c-Jun concentration was variable in order to determine the affinity of VRK1 for c-Jun. VRK1 has an affinity for c-Jun, with a $\mathrm{Km}$ of $0.4 \mu \mathrm{M}$ (Figure $2 \mathrm{~b}$ ), which is well suited for the intracellular concentrations of the c-Jun protein. The strong signal of the GST-VRK1 protein is due to the high autophosphorylation activity of this kinase (LopezBorges and Lazo, 2000; Barcia et al., 2002).

Next, it was determined if SP600125, a very potent inhibitor of JNK kinase activity (Bennett et al., 2001; Han et al., 2001), could affect VRK1 kinase activity. In an in vitro kinase assay, a wide range of SP600125 concentrations were used (Figure 2c). VRK1 was not affected even by concentrations that were up to two orders of magnitude higher than the one, $\mathrm{IC}_{50}=0.09 \mu \mathrm{M}$, required for $\mathrm{JNK}$ inhibition (Bennett et al., 2001; Han et al., 2001). Therefore, we can conclude that the activity of VRK1 is not sensitive to the SP600125 inhibitor (Figure 2c), since it does not inhibit the autophosphorylation or the phosphorylation of cJun. VRK1 protein with the K179E substitution in the active site has no kinase activity on itself nor on c-Jun (Figure 2c). 
8952

a

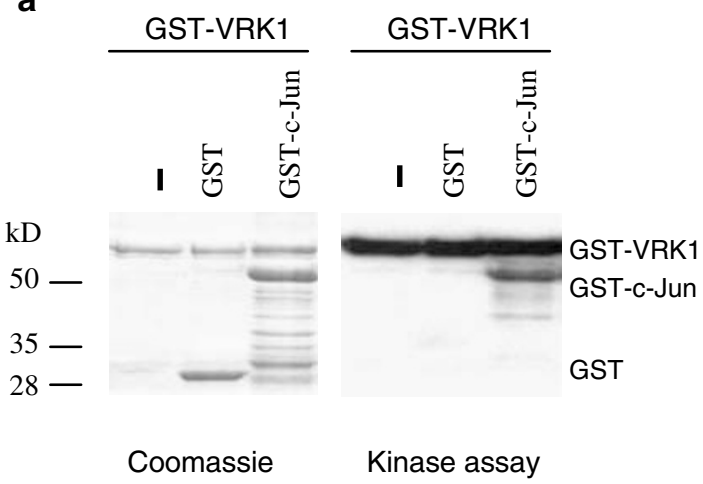

b
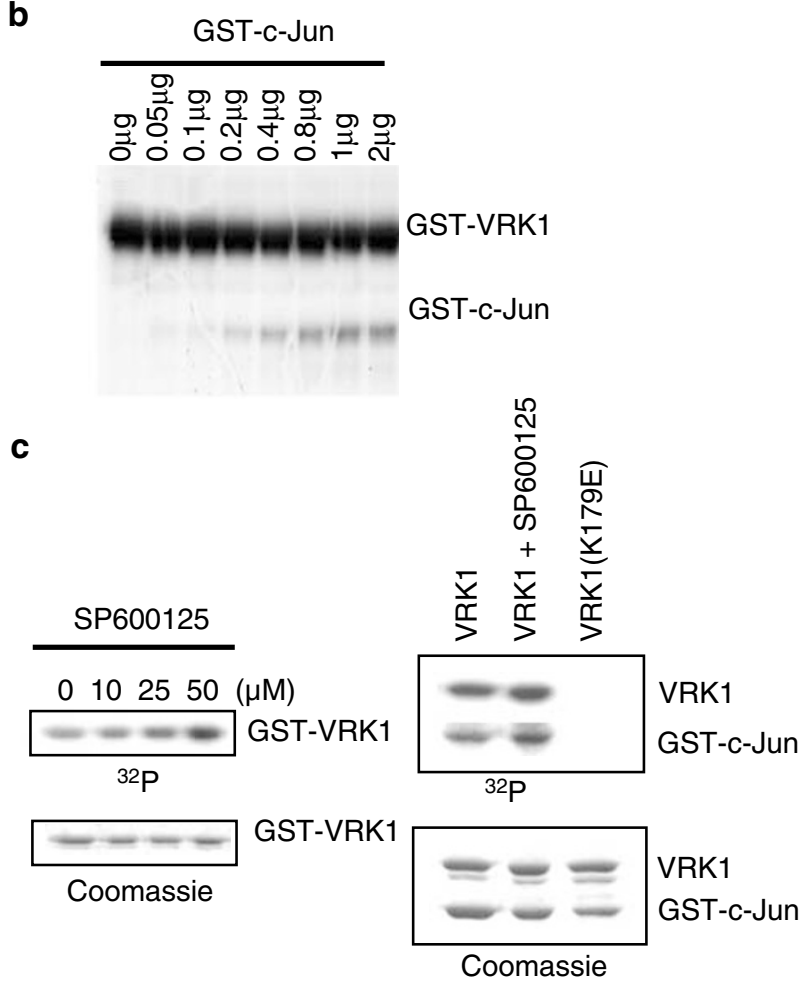

Figure 2 (a) Phosphorylation of the N-terminus of c-Jun by the human VRK1 protein. Both proteins were used as GST-fusion proteins in an in vitro kinase assay. At the completion of the reaction the samples were loaded in an SDS-polyacrylamide gel. In the left panel is the Coomassie blue staining and in the right panel the incorporation of radioactivity is shown. (b) Kinetics of VRK1 phosphorylation of the c-Jun protein. As substrate, a GST-c-Jun fusion protein was used in the assay and its concentration was changed as indicated, the quantification was performed in the linear response range of all parameters. (c) Effect of different concentrations of SP600125 on the autophosphorylation activity of VRK1 (left panel) or lack of effect of $25 \mu \mathrm{M}$ SP600125 on the phosphorylation of GST-c-Jun (right panel). Also is included an assay with the inactive mutant VRK1 (K179E) to show its lack of activity (right panel). Both incorporation of radioactivity and the amount of protein in the gel (Coomassie) are shown

\section{c-Jun is phosphorylated in Ser63 and Ser73 by VRK1}

To identify the residues phosphorylated by VRK1 in the c-Jun molecule we performed a phosphoaminoacid a

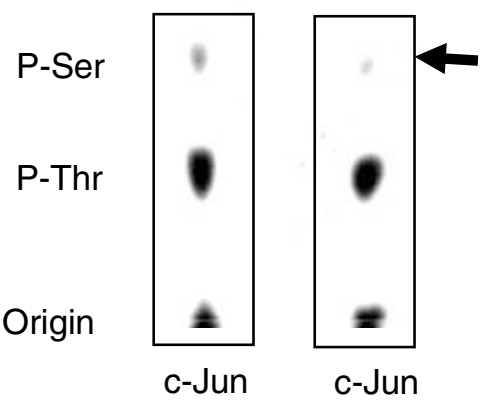

(Ser63,73Ala)

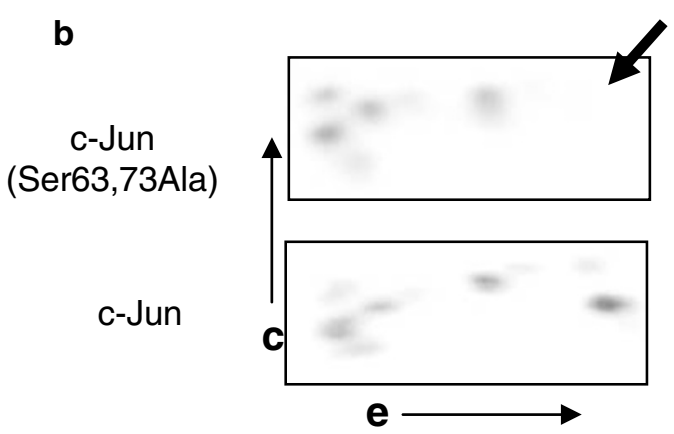

Figure 3 (a) Phosphoaminoacid analysis of the wild-type GST-cJun and mutant GST-c-Jun (Ser 63,73 Ala). The intensities of the spots were quantified in a phosphorimager system. The arrow indicates the loss of serine phosphorylation in the c-Jun double mutant. (b) Phosphopeptide analysis of GST-c-Jun and mutant GST-c-Jun (Ser 63, 73 Ala), first dimension is a high-voltage electrophoresis and the second dimension is a thin layer chromatography. The arrow indicates the spot that disappears in the double c-Jun mutant

analysis using as substrate the GST-c-Jun fusion protein and its mutant form where Ser63 and Ser73 have been replaced by alanine. Both Ser and Thr residues are phosphorylated (Figure 3a). The incorporation of radioactivity in the phospho-Ser position falls by more than $80 \%$ when the Jun Ser 63,73 Ala mutant was used, suggesting that these residues are likely to be the main targets for VRK1 phosphorylation. In addition, there is a strong phosphorylation of Thr residues in the c-Jun molecule as shown by the spot corresponding to phospho-Thr (Figure 3a). In order to identify the phosphorylated residues we also performed a phosphopeptide map of the phosphorylated GST-c-Jun and of its double mutant containing the S63A and S73A substitutions, which are the residues targeted by JNK. The phosphopeptide of the c-Jun double mutant, S63A and S73A, resulted in the loss of a peptide (Figure 3b); however, there were still several other phosphorylated peptides, which accounted for the lack of total loss of signal in the phosphoaminoacid analysis. These additional peptides are likely to result from the phosphorylation in $\mathrm{Thr}$ residues, although some additional phosphorylation in other Ser residues cannot be excluded. 


\section{VRK1 stabilizes c-Jun}

The phosphorylation of c-Jun by other kinases, such as $\mathrm{JNK}$, resulted in an increase in its stability and consequently in an increase in the intracellular levels of the c-Jun protein, because the phosphorylated molecules can dimerize, preventing their binding to ubiquitin ligase (Fuchs et al., 2000). Therefore, we performed an experiment to test if VRK1 could also induce a c-Jun accumulation as a result of its phosphorylation. For this aim 293T cells were transfected with pHA-VRK1 and a fixed amount of pHA-c-Jun. The c-Jun and VRK1 levels were determined by immunoblots with an anti-HA antibody (Figure 4a). EGFP protein under the promoter of CMV was also transfected and the levels of EGFP were determined by Western blot. The levels of EGFP were not affected by increasing the amount of transfected VRK1, ruling out the effect on the CMV plasmid promoter (Figure $4 \mathrm{a}$ ).

To further demonstrate the specificity of this accumulation, similar experiments were performed using an inactive kinase by introducing a specific substitution in the catalytic site, the VRK1 (K179E) mutant. When this inactive kinase was used there was a loss of the c-Jun accumulation (Figure 4b), suggesting that it was a consequence of the phosphorylation by this kinase.

To confirm that the VRK1 effect is associated with cJun phosphorylation, the phosphorylation of the endogenous c-Jun protein in residue Ser63 was determined, with a specific antiphospho-specific antibody, in 293T cell transfected with increasing amounts of VRK1. As the amount of VRK1 was increased so did the phosphorylation of the endogenous c-Jun in Ser63 (Figure 4c).

\section{VRK1 stimulates c-Jun dependent transcription}

Owing to the phosphorylation of residues Ser63 and Ser73 in the c-Jun molecule, which are identical to those phosphorylated by the c-Jun $\mathrm{N}_{2}$-terminal kinase (JNK), it is very likely that the VRK1 activity will also modulate c-Jun dependent transcription. To study the possible effect of VRK1 on the transcriptional activity

Figure 4 (a) Accumulation of c-Jun by increasing the amount of transfected HA-VRK1 (pCMV-HA-hVRK1) in 293T cells; the control protein GFP expressed under the same promoter is not affected by increasing concentrations of the kinase. 293T cells $\left(1.5 \times 10^{6}\right)$ were transfected with $1 \mu \mathrm{g}$ of HA-c-Jun and increasing amounts of the HA-VRK1 kinase (pCMV-HA-hVRK1). The amount of DNA in the transfection was kept constant by the addition of empty expression vector. (b) The inactive kinase VRK1 (K179E) does not induce the accumulation of c-Jun. At the bottom the quantification and relative effect of the kinase, wild-type or inactive mutant (K179E), on the accumulation of c-Jun are shown. Quantification was performed in the linear response range of all signals. $\beta$-Actin was used as a gel load control. (c) The phosphorylation of the endogenous c-Jun in Ser63 increases with increasing amounts of transfected VRK1 ((pCMV-HA-hVRK1) Phosphorylation of c-Jun phosphorylated in Ser63 was detected with an antibody specific for this phosphorylation from Cell Signaling. The control are cells transfected with empty vector of c-Jun, we tested the effect by transient transfection in NIH-3T3 cells with different combinations of kinases and c-Jun substrates (wt or mutant), in a reporter system consisting in the fusion of the N-terminus of c-Jun with a GAL4 DNA binding domain (c-Jun-GAL), and a luciferase reporter vector $5 \times$ GAL4-luciferase. The reporter construct was activated when recognized by dimers of the DNA binding domain, which only occurs when the c-Jun moiety of the fusion protein was phosphorylated. First, a dose-response analysis was
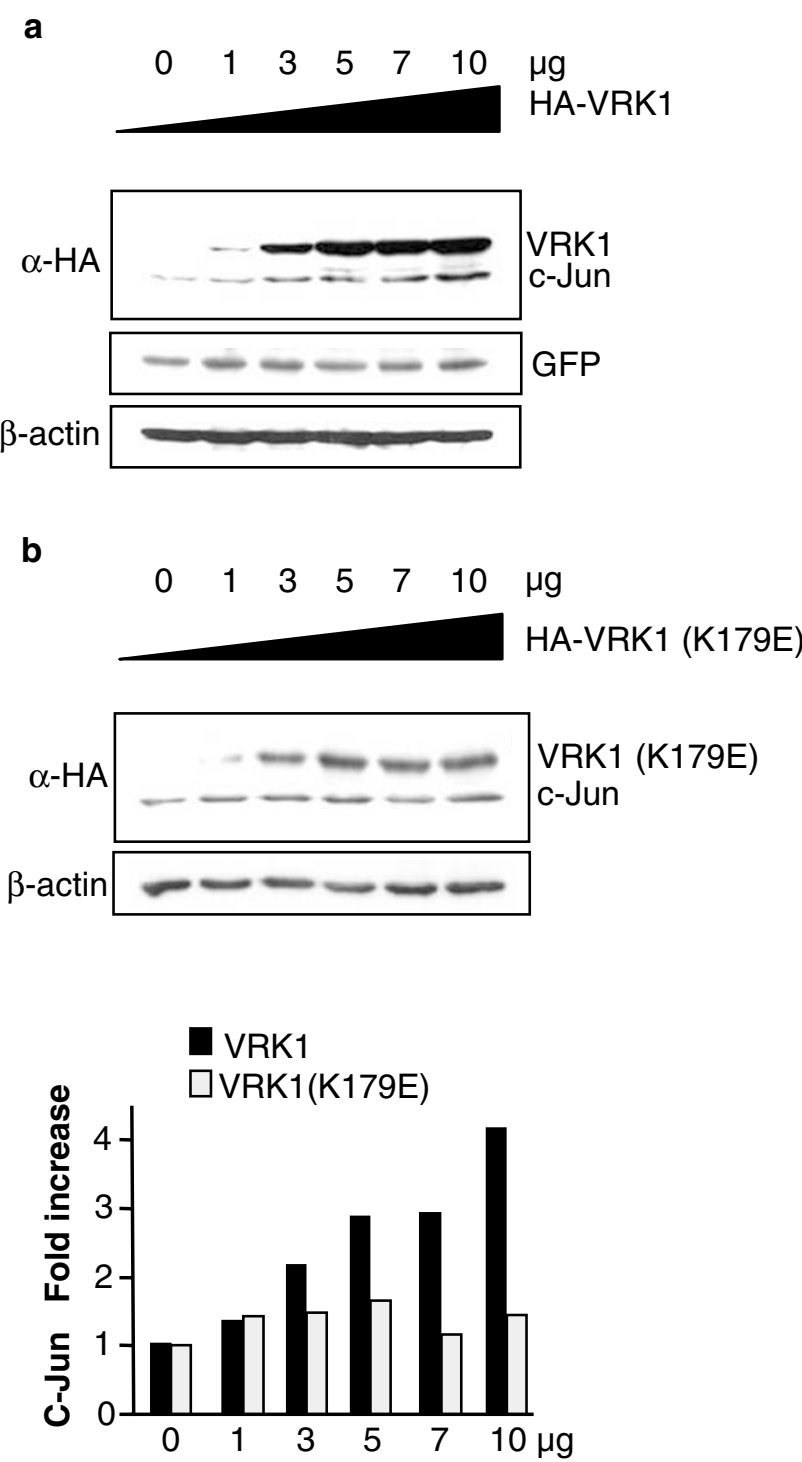

C

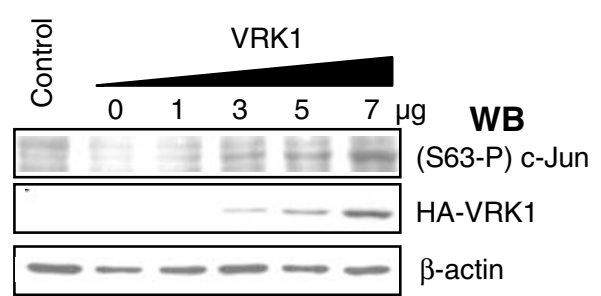


performed in $293 \mathrm{~T}$ cells using different amounts of pHA-VRK1 plasmid. As the VRK1 protein kinase was increased, so was the activation of transcription (Figure 5a). Next, the effect was also studied in NIH 3T3 fibroblasts, and the VRK1 effect on transcription was compared with that of JNK. In cells transfected with HA-JNK as positive control, there was a very important increase in transcriptional activity. The transfection with the HA-VRK1 kinase also induced

a

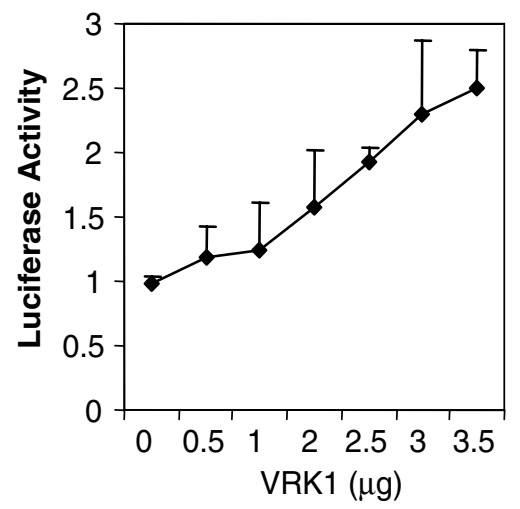

b

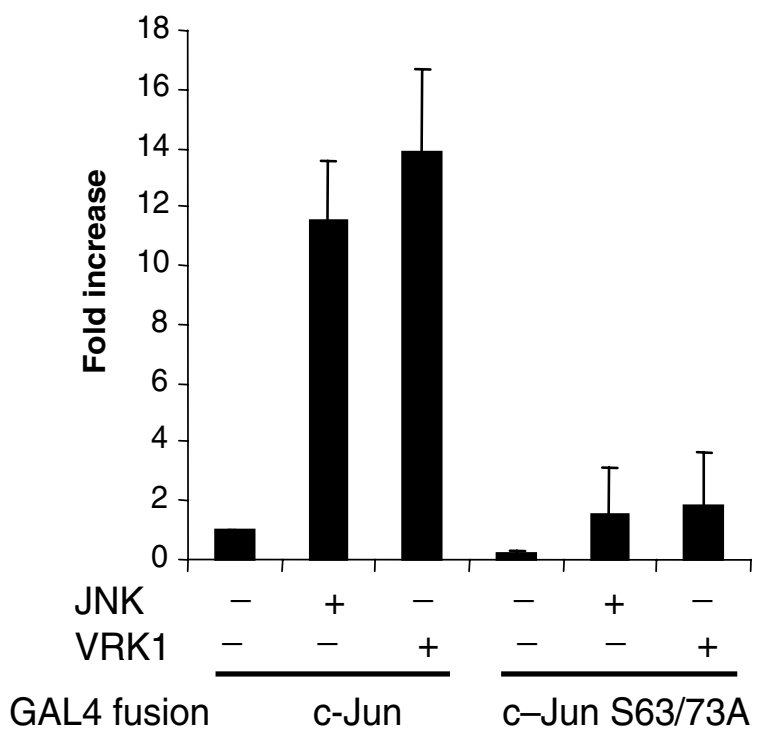

Figure 5 (a) Dose-response analysis of transcription activation by VRK1 using a dimerization-dependent assay in transfected 293T cells $\left(5 \times 10^{5}\right.$ cells per plate). (b) VRK1 phosphorylation induces the transcriptional activity of c-Jun. NIH 3T3 fibroblast were transfected with the control plasmid or with either $3 \mu \mathrm{g}$ of HAJNK (pHA-JNK) or $3 \mu \mathrm{g}$ of HA-VRK1 (pCMV-HA-hVRK1). In the reporter system the following plasmids were used as indicated, $1 \mu \mathrm{g}$ of $5 \times$ GAL4-Luc (reporter), and $1 \mu \mathrm{g}$ of GAL4-c-Jun (1-257) or $1 \mu \mathrm{g}$ of GAL4-c-Jun (1-257, S63/73A) expression vectors encoding for fusion proteins containing the GAL4 DNA binding domain and the c-Jun activation domain (residues 1-257) in wild type and mutant forms (not phosphorylatable by substitutions of serine 63 and 73 by alanine). For normalization of the transfection efficiency $0.5 \mu \mathrm{g}$ of the plasmid pRL-tk, Renilla luciferase reporter, was included in the transfection. The total amount of DNA in the transfection was kept constant at $5.5 \mu \mathrm{g}$ by the addition of empty vector. Three independent experiments were performed an activation of transcription, which was similar to that induced by JNK (Figure 5b). The transcriptional activation of c-Jun by either HA-JNK or the HAVRK1 was completely blocked when the cells were transfected with the mutant form of c-Jun containing the double substitution of Ser63 and Ser73 for Ala, as shown in Figure 5b. Therefore, we can conclude that VRK1 acts as a potent activator of c-Jun transcriptional activity by phosphorylation of the same residues as those phosphorylated by JNK.

\section{VRK1 interacts with c-Jun but not JNK}

It is possible that the effect of VRK1 might be mediated directly by the interaction of VRK1 with cJun or indirectly via JNK. To test this possibility 293T cells were transfected with expression plasmids pHA-cJun or pHA-JNK in combination with a construct expressing pCDNA-VRK1-myc. The transfected cells express very well all the constructs, as shown by Western blots of the corresponding transfected cells (Figure 6a). These extracts were used for reciprocal immunoprecipitation and immunoblots (Figure 6b). In cells transfected with HA-c-Jun and VRK1-myc, the immunoprecipitation with anti-HA brings down VRK1 as detected in the immunoblot. The inverse situation, a

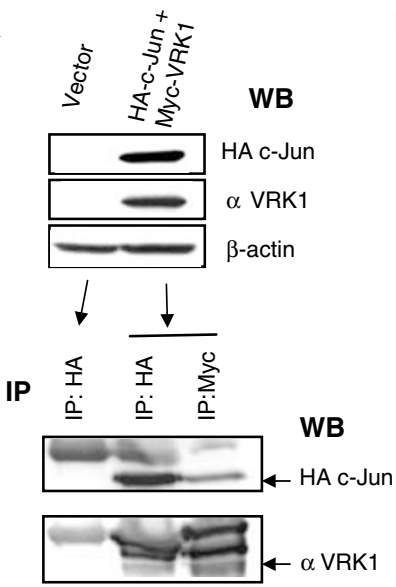

b

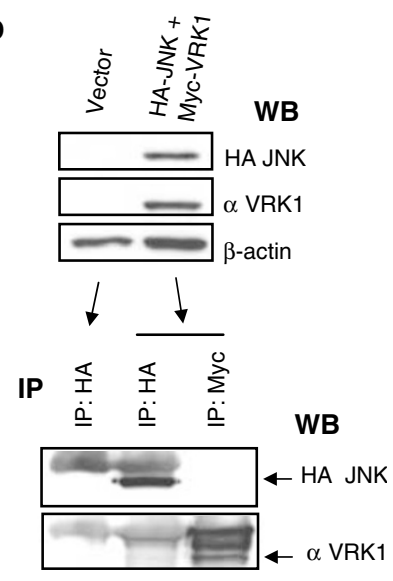

Figure 6 Interaction of VRK1 with c-Jun (a) or JNK (b). At the top, the immunoblot of whole-cell extracts of cells $\left(1.5 \times 10^{6}\right.$ cells dish) transfected with either the combination of $4 \mu \mathrm{g}$ of VRK1 (pCDNA-hVRK1-Myc) and $4 \mu \mathrm{g}$ of pHA-c-Jun (A top) or $4 \mu \mathrm{g}$ of VRK1 (pCDNA-hVRK1-Myc) and $4 \mu \mathrm{g}$ of HA-JNK (pHA-JNK) (b top) is shown. The transfected proteins were detected by immunoblot with a commercial antibody against the specific tag (HA epitope) or a polyclonal antibody specific for VRK1 (top panels). These extracts were used for the reciprocal immunoprecipitation followed by immunoblot (WB) of cells transfected with VRK1 (pCDNA-hVRK1-Myc) and pHA-c-Jun in part (a) of the figure, in both immunoprecipitates the other protein is detected (a, bottom). The reciprocal immunoprecipitation followed by immunoblot from cells transfected with VRK1 (pCDNA-hVRK1-Myc) and pHA-JNK (b, bottom) was also performed. In these immunoprecipitates, the second protein is not detected by Western blot, suggesting VRK1 and JNK do not interact. Immunoprecipitations were performed with commercial antibodies specific for the HA or Myc epitopes, and the Western blots were performed with either a commercial antibody for the HA epitope or a polyclonal antibody specific for the VRK1 protein 
in the immunoprecipitate of VRK1, c-Jun is also detected with the anti-HA antibody (Figure 6b, left). However, when the VRK1 and JNK combination was used immunoprecipitation with each of them did not bring down the other as shown in the immunoblots (Figure 6b, right). These data suggest that VRK1 can interact with c-Jun, but not JNK. However the complex is likely to be a minor fraction of the total protein expressed in the cells, since only the c-Jun fraction located in the nucleus can interact with VRK1.

\section{VRK1 cooperates with JNK activation of c-Jun}

The utilization by VRK1 and JNK of the same residues as targets in the c-Jun molecule suggested that perhaps both kinases could cooperate in c-Jun regulation. To test if the activities of JNK and VRK1 have an additive effect, suboptimal concentrations of JNK 1 and VRK1 were selected, such as 0.4 or $0.8 \mu \mathrm{g}$ of each kinase expression plasmid, and used for transfection of 293T cells. As shown in Figure 7, the suboptimal concentration of each kinase by itself induces a moderate increase in c-Jun dependent transcription. However, the mixture of the two kinases induced an additive effect, which is dependent on the amount of kinase transfected. Therefore, we concluded that both kinases can activate c-Jun in a situation where there is no maximal activation of the transcriptional activity of c-Jun.

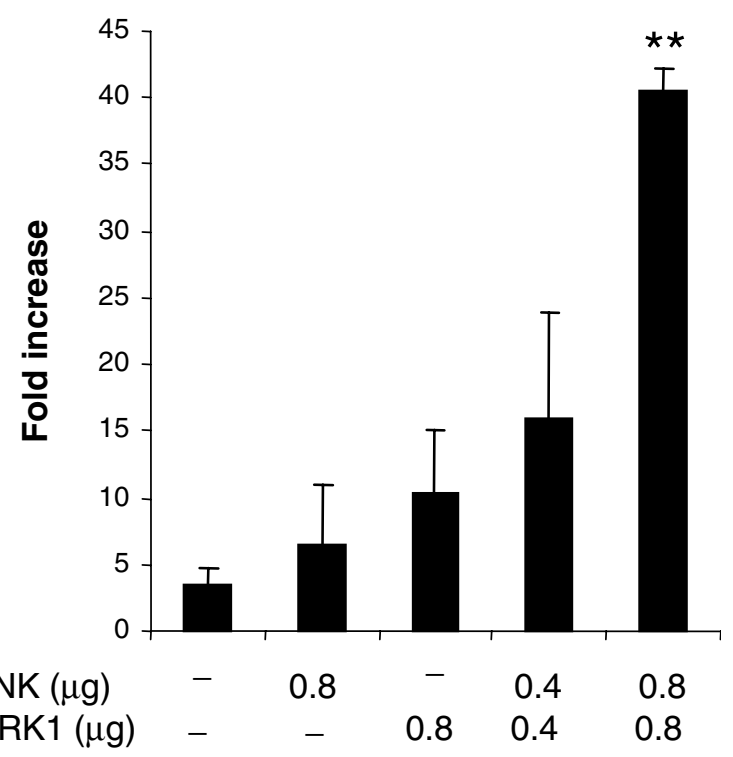

Figure 7 Cooperation between JNK and VRK1 in c-Jun activation. 293T cells were transfected with different amounts of HA-JNK (pHA-JNK) or HA-VRK1 (pCMV-HA-hVRK1) by themselves or in combination using as reporter system $5 \times$ GAL4Luc $(1 \mu \mathrm{g})$, GAL4-c-Jun $(1 \mu \mathrm{g})$ and $0.5 \mu \mathrm{g}$ of the plasmid pRL-tk. Activation of c-Jun by either transfected HA-JNK or HA-VRK1; and additive effect of suboptimal concentrations of HA-JNK (pHA-JNK) and HA-VRK1 (pCMV-HA-hVRK1) on the transcriptional activity of c-Jun. Mean with s.d. from three independent experiments are shown. ${ }^{* *} P<0.01$

\section{Discussion}

The phosphorylation of the c-Jun transcription factor by the nuclear VRK1 protein may represent a novel pathway of c-Jun regulation. The VRK1 kinase is not integrated in any known pathway and is not included in the MAPK kinase signaling complexes with the JIP-1 proteins. These complexes are formed in the cytoplasm, while VRK1 is located in the nucleus. This suggests that while the MAPK kinase complexes are implicated mainly in response to extracellular signals, the pathway mediated by VRK1 might be a response mechanism to events occurring within the nuclei, which may include, for example, sensor mechanisms of DNA damage. However, the upstream regulatory elements of VRK1 are not yet known. In this context, it is interesting to note that two of the proteins phosphorylated by VRK1, p53 and c-Jun, are components of the cellular mechanisms implicated in the response to different types of stress (Figure 8).

The affinity of VRK1 for the c-Jun protein is well suited to respond to fluctuations in its intracellular concentration. Thus, if by any stimuli the level of c-Jun protein increases in the nuclei, it will be phosphorylated and thus become transcriptionally active. The phosphorylation of c-Jun in Ser63 and Ser73 suggest that these residues can be phosphorylated independently of the c-Jun activation by JNK, as shown by the additive effect on c-Jun transcriptional activation by JNK and VRK1. Furthermore, both kinases can reach a similar level of maximal activation of c-Jun transcriptional

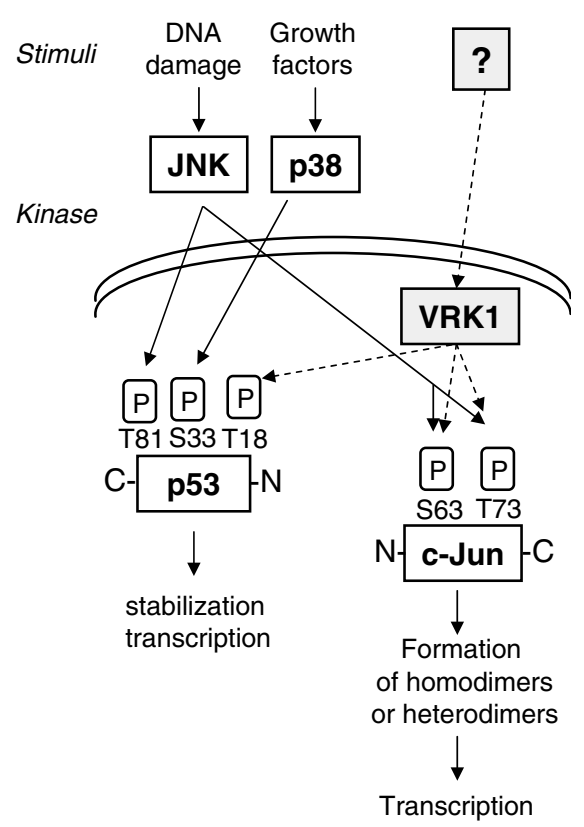

Figure 8 Diagram illustrating signaling pathways and the targets of VRK1 and signals converging on c-Jun. VRK1 forms a complex with nonphosphorylated c-Jun in the nucleus and therefore represent a subpopulation of the total c-Jun protein in the cell. JNK binds to c-jun in the cytosol and the complex is translocated to the nucleus where they dissociate, but this c-Jun that has been phosphorylated by JNK cannot be a target for VRK1 
activity, indicating that the VRK1 pathway is likely to be independent of $\mathrm{JNK}$, but at least part of its transcriptional effects might be the same. There is an important functional difference between JNK and VRK1. JNK is activated in the cytosol and forms a stable complex with c-Jun that is translocated to the nuclei. On the other hand, VRK1 is already in the nuclei and its substrate is only the fraction of c-Jun molecules located within the nuclei. This fact can explain that the amount of c-Jun forming a stable complex is small in the VRK1 immunoprecipitate, since it represents a subpopulation of c-Jun molecules within the nucleus. Therefore, there is a compartmentalization regarding the interaction between the kinase and its substrate. In those situations where the JNK-c-Jun complex is already formed in the cytosol and translocated to the nucleus, it is unlikely that there could be an additional effect of VRK1 since JNK would physically prevent the interaction of VRK1 with c-Jun, or the c-Jun is already phosphorylated. The additive effect of c-Jun phosphorylation by VRK1 and JNK suggests two potential consequences of phosphorylation. One is that the two pathways can activate c-Jun in an additive manner if the activation occurs at suboptimal doses. The alternative would be that maximal activation by one pathway will prevent activation by the second pathway. In this latter case the biological differences will be determined by the different substrate specificity of the kinases involved.

Most studies on c-Jun regulation have been performed under catastrophic or massive stress conditions that are unlikely to represent its biological selection during evolution, since they are stress of a type or magnitude never detected by normal cells such as irradiation at very high doses or chemotherapeutic drugs. The magnitude of normal damage is much lower and suboptimal, and that is the one that participated during evolution; therefore, c-Jun must have a much more subtle regulation, and VRK1 might be one of these regulatory elements. c-Jun is required for normal cell proliferation, and under severe stress conditions an apoptotic reaction is often started (Dunn et al., 2002). In the absence of stress stimulation, perhaps the role played by VRK1 is that of maintaining a basal activity of AP1 dependent transcription that requires c-Jun as part of the transcription factor dimer. However, the fine tuning of these mechanism and their implication in oncogenesis are far from being well characterized, suggesting c-Jun regulation is more complex than originally realized (Kennedy and Davis, 2003). Alteration in c-Jun levels have been detected in several tumor types, changes that might modify the expression of other genes and be oncogenic (Wodrich and Volm, 1993; Tiniakos et al., 1994; Neyns et al., 1996; Szabo et al., 1996; Aoyagi et al., 1998; Antonyak et al., 2002; Volm et al., 2002; Meggiato et al., 2003).

In this report we have demonstrated that VRK1 can regulate c-Jun dependent transcriptional activity, which can cooperate with other kinases, such as JNK. These results are a contribution to the elucidation of the potential roles of the VRK1 protein in cell biology, representing an additional step in the characterization of a new signaling pathway.

\section{Materials and methods}

\section{Fusion proteins}

The GST-c-Jun (1-223) fusion protein and its double mutant with the Ser63 and 73 residues substituted by Ala were a gift of M Karin (San Diego, CA, USA). The GST-VRK1 fusion protein has been previously described (Lopez-Borges and Lazo, 2000).

\section{Plasmids}

The human VRK1 protein was expressed from constructs pCDNA-VRK1-Myc or pCMV-HA-hVRK1, and pCMVHA-hVRK1 (K179E) that have the coding VRK1 sequence tagged with the Myc or HA epitopes. The exogenous kinase was pHA-JNK from S Gutkind (NIH, Bethesda, MD, USA). Plasmid pCMV-HA-c-Jun was from D Bohmann (EMBL, Heidelberg, Germany). For reporter assays of luciferase activity the following plasmids from M Karin (University of California, San Diego) were used, the $5 \times$ GAL4-Luc (reporter), and GAL4-c-Jun (pSG424 Gal4-c-Jun, residues 1257) and GAL4-c-Jun S63/73A (pSG424 Gal4-c-Jun S63/ 73A, residues 1-257) expression vectors encode fusion proteins containing the GAL4 DNA binding domain and the c-Jun activation domain in wild type and mutant forms (not phosphorylatable by mutation of both serines 63 and 73 to alanine). As internal control in transcription assays we used the pRL-tk plasmid from Promega (Madison, WI, USA).

\section{Kinase activity assay}

Kinase activity was determined by assaying protein phosphorylation in a mixture containing $20 \mathrm{mM}$ Tris- $\mathrm{HCl}, \mathrm{pH} 7.5,5 \mathrm{~mm}$ $\mathrm{MgCl}_{2}, 0.5 \mathrm{mM}$ dithiothreitol, $150 \mathrm{mM} \mathrm{KCl}$ and $5 \mu \mathrm{M}(5 \mu \mathrm{Ci})$ $\left[\gamma-{ }^{32} \mathrm{P}\right] \mathrm{ATP}, 5 \mu \mathrm{g}$ of GST-VRK1 protein and $10 \mu \mathrm{g}$ of another protein as substrate when indicated. The reaction was routinely performed for $30 \mathrm{~min}$ at $30^{\circ} \mathrm{C}$ (Lopez-Borges and Lazo, 2000; Barcia et al., 2002). The specific inhibitor for JNK, SP600125 was from Sigma. For the kinetic analysis the reaction conditions were similar with the exception that the amount of GST-c-Jun varied as indicated in the corresponding experiment. Always the reaction was performed in the linear response range of incorporation of radioactivity. Quantitation was performed by two different techniques, direct quantitation in a BioRad personal phosphorimager or by exposure to X-ray film in the linear response range and quantitation of the bands by densitometry. With both methods the same results were obtained, where the Vmax was always determined in arbitrary units as pixels per surface area unit.

\section{Acid hydrolysis and phosphoaminoacid analysis}

An in vitro kinase assay was performed with GST-VRK1 and the GST-c-Jun or GST c-Jun S63/73A as substrates. The phosphorylated proteins were fractionated by an SDS-polyacrylamide electrophoresis and transferred onto an Immobilon-P filter (Millipore, Bedford, MA, USA) and the radioactive bands corresponding to GST-c-Jun and GST cJun S63/73A were excised and the protein was eluted. The eluted protein was hydrolyzed with $200 \mu \mathrm{l}$ of $6 \mathrm{~N} \mathrm{HCl}$ at $110^{\circ} \mathrm{C}$ for $2 \mathrm{~h}$. The sample was freeze-dried and resuspended in a mixture containing $2 \mu \mathrm{l}$ of a stock solution at $1 \mathrm{mg} / \mathrm{ml}$ of P-Ser, P-Thr and P-Tyr as reference markers, $1 \mu \mathrm{l}$ of $1 \%$ bromophenol blue and $2 \mu \mathrm{l}$ of formic acid/acetic acid/water $(1: 3.1: 35)$ mix at $\mathrm{pH}$ 1.9. The mixture was applied to a cellulose chromatoplaque (Merck, Darmstadt, Germany) and subject to 
flat bed electrophoresis in a Multiphor II system (Amersham Biosciences, Little Chalfont, UK) at $1.5 \mathrm{kV}$ for $90 \mathrm{~min}$. After air drying, the radioactivity on the chromatoplaque was analyzed with an FX Personal Imager (BioRad, San Diego, CA, USA) or exposed to X-ray film. The phosphoaminoacid markers were detected by staining with a $0.25 \%$ ninhydrin solution in acetone.

\section{Tryptic phosphopeptide map analysis}

GST-c-Jun and GST c-Jun S63/73A phosphorylated proteins were isolated from an Immobilon- $P$ membrane (Millipore). The band with the phosphorylated protein was excised, incubated with $0.5 \%$ polyvinyl-pyrrolidone in $100 \mathrm{mM}$ acetic acid for $30 \mathrm{~min}$ at $37^{\circ} \mathrm{C}$, followed by extensive washes with water and fresh $50 \mathrm{~mm} \mathrm{NH}_{4} \mathrm{HCO}_{3}$. The membrane was incubated twice, with $10 \mu \mathrm{g}$ of trypsin (Roche Diagnostics, Mannheim, Germany) in $50 \mathrm{~mm} \mathrm{NH} \mathrm{NHCO}_{3}, \mathrm{pH} 8.0$ for $2 \mathrm{~h}$ at $37^{\circ} \mathrm{C}$, and a third time for $18 \mathrm{~h}$. The membrane was extensively washed with sterile distilled water. The washes were freezedried, and oxidized with 9:1 performic acid/hydrogen peroxide for $1 \mathrm{~h}$ at room temperature, followed by freezedrying. The sample was resuspended in $5 \mu \mathrm{l}$ of formic acid: acetic acid: water $(1: 3.12: 35.88) \mathrm{pH} 1.9$ and applied to a chromatoplaque (Merck, Darmstadt, Germany). The first dimension was an electrophoresis at $1.5 \mathrm{kV}$ for $90 \mathrm{~min}$ in a Multiphor system (Pharmacia, Uppsala, Sweden). The chromatoplaque was left to air dry, and turned $90^{\circ}$ for the second dimension. The second dimension was a chromatography performed in acetic acid:pyridine: n-butanol: water $(1: 3.33: 5: 4)$ for $13 \mathrm{~h}$. The plaque was left to air dry and radioactivity was detected using an X-ray film or by a phosphorimager system (BioRad, San Diego, USA)

\section{Cell lines and transfections}

NIH 3 T3 and 293T human embryo kidney cells were grown in Dulbecco's modified Eagle's medium (DMEN) supplemented with 10\% fetal bovine serum (FBS), 1\% L-glutamine and $1 \%$ penicillin-streptomycin at $37^{\circ} \mathrm{C}$ in a humidified atmosphere of $5 \% \mathrm{CO}_{2}$. Transfections were performed by the calcium phosphate method or with JetPEI reagent (Polytransfection, Illkirch, France), according to the manufacturer's recommendations. The total amount of DNA within the experiments was kept constant by adding the respective empty vector plasmid DNA to the transfection mixtures. In experiments using $293 \mathrm{~T}$ cells, usually $1.5 \times 10^{6}$ cells were plated in a P100 dish, or $5 \times 10^{5}$ in a P60 dish $16 \mathrm{~h}$ prior to transfection. For NIH $3 \mathrm{~T} 3$ cells, $3 \times 10^{5}$ cells were seeded in a P60 dish.

\section{Immunoblots}

Whole-cell extracts were collected $48 \mathrm{~h}$ post-transfection. The cell monolayer was washed twice in PBS and the cells were lysed by incubation in RIPA buffer $(150 \mathrm{mM} \mathrm{NaCl}, 1.5 \mathrm{~mm}$ $\mathrm{MgCl}_{2}, 10 \mathrm{mM} \mathrm{NaF}, 10 \%$ glycerol, $4 \mathrm{mM}$ EDTA, $1 \%$ Triton $\mathrm{X}-100,0.1 \%$ SDS, $1 \%$ Deoxycholate, $50 \mathrm{mM}$ HEPES, pH 7.4), plus $1 \mathrm{mM} \mathrm{Na} \mathrm{VO}_{4}, 10 \mu \mathrm{g} / \mathrm{ml}$ leupeptin, $10 \mu \mathrm{g} / \mathrm{ml}$ aprotinin and $1 \mathrm{mM}$ PMSF for $30 \mathrm{~min}$ on ice and precleared by centrifugation at 13000 r.p.m. for $20 \mathrm{~min}$ at $4{ }^{\circ} \mathrm{C}$. The extracts were analyzed by SDS-PAGE under denaturing conditions and transferred onto Immobilon-P membranes (Millipore, Bedford, MA, USA). The membranes were blocked with 5\% skimmed milk in TBS-T, and then incubated with the specific antibody, antiHA at 1:1000 dilution (Covance, Berkeley, CA, USA) or a rabbit anti-VRK1 polyclonal antibody at 1:500 dilution. The c-Jun phosphorylated in Ser63 was detected with a specific antibody from Cell Signaling at a 1:1000 dilution (Beverly, MA, USA) after blocking the membrane with $1 \%$ BSA. As secondary antibody a rabbit anti-mouse IgG or a goat antirabbit $\mathrm{IgG}$ with peroxidase were used at a dilution of $1: 10000$ and the blot was developed with an ECL chemiluminescence kit from Amersham Bioscience.

\section{Immunofluorescence and confocal microscopy}

HeLa cells were grown on uncoated glass coverslips introduced into $100-\mathrm{mm}$ plates $(150000$ cells/plate $)$. At $30 \mathrm{~h}$ posttransfection using JetPEI (Polytransfection, France), cells were rinsed twice in PBS, fixed with $3.7 \%$ paraformaldehyde in PBS for $15 \mathrm{~min}$ at room temperature, permeabilized by incubation with PBS containing $0.5 \%$ Triton X-100 for $15 \mathrm{~min}$, and were then treated with $0.1 \mathrm{M}$ glycine in PBS for $15 \mathrm{~min}$. Nonspecific sites were blocked by incubation with PBS containing $1 \%$ BSA for $30 \mathrm{~min}$ at room temperature. Cells were then washed in PBS containing $0.05 \%$ Tween-20 for $5 \mathrm{~min}$ and incubated with the primary antibodies diluted in PBS for $1 \mathrm{~h}$ at room temperature. After that, cells were incubated with one of the following secondary antibodies: antimouse secondary antibody coupled to Cy2 (Sigma) $(1: 200$ dilution), anti-rabbit secondary antibody coupled to $\mathrm{Cy} 3$ (Sigma) (1:200 dilution). Fluorescence images were captured with a Zeiss LSM 510 confocal microscope. Fluorochromes were excited using an ${ }^{18}$ Argon laser (488 $\mathrm{nm}$ excitation wavelength) for $\mathrm{Cy} 2$ and an ${ }^{2} \mathrm{He} /{ }^{10} \mathrm{Ne}$ laser (543 nm excitation wavelength ) for $\mathrm{Cy} 3$.

\section{Transcriptional assays}

Reporter gene assays were performed with the Dual-Luciferase reporter assay system (Promega) in cell extracts prepared at $36-48 \mathrm{~h}$ after transfection. The assay is based on the use of $\mathrm{p}$ SG-424-c-Jun or pSG-424-c-Jun S63/73A constructs with a GAL-4-DNA binding domain and a p5 $\times$ GAL4-Luc reporter that were cotransfected in each sample in combination with pRL-tk Renilla luciferase as an internal control for transfection efficiency. The total amount of DNA within the experiments was kept constant by adding the respective empty vector plasmid DNA to the transfection mixtures. Luciferase activity was determined with $20 \mu \mathrm{l}$ of total cell extract using the Dual Luciferase reporter assay kit from Promega (Madison, WI, USA). The generated light was detected with an OPTOCOM-1 luminometer (MGM Instruments, Inc., Hamden, CT, USA) as previously described (Hernandez-Torres et al., 2001; Yunta et al., 2002). The activity of the reporter luciferase was expressed relative to the activity in control vector transfected cells. Transcription reporter experiments were performed in triplicate. All results were analyzed by comparing with the control (unpaired Student's test) and in the figures are shown the mean \pm s.d. of independent triplicate cultures as well as the $P$-value.

\section{Acknowledgements}

AS and CS have predoctoral fellowships from Consejo Superior de Investigaciones Científicas and the Fundação para a Ciência e a Tecnologia (Portugal) respectively. This work was funded by grants from Ministerio de Educación y Ciencia (SAF2000-0169, SAF2004-2900), Fondo de Investigación Sanitaria (FIS02/0585), Junta de Castilla y LeónConsejerías de Educación (CSI18-03) and Sanidad (SA 01/ 02), Fundación de Investigación Médica MMA and Fundación Memoria Samuel Solórzano Barruso. 


\section{References}

Adler V, Fuchs SY, Kim J, Kraft A, King MP, Pelling J and Ronai Z. (1995). Cell Growth Differ., 6, 1437-1446.

Antonyak MA, Kenyon LC, Godwin AK, James DC, Emlet DR, Okamoto I, Tnani M, Holgado-Madruga M, Moscatello DK and Wong AJ. (2002). Oncogene, 21, 5038-5046.

Aoyagi K, Shima I, Wang M, Hu Y, Garcia FU and Stearns ME. (1998). Clin. Cancer Res., 4, 2153-2160.

Barcia R, Lopez-Borges S, Vega FM and Lazo PA. (2002). Arch. Biochem. Biophys., 399, 1-5.

Bennett BL, Sasaki DT, Murray BW, O’Leary EC, Sakata ST, $\mathrm{Xu}$ W, Leisten JC, Motiwala A, Pierce S, Satoh Y, Bhagwat SS, Manning AM and Anderson DW. (2001). Proc. Natl. Acad. Sci. USA, 98, 13681-13686.

Chang L and Karin M. (2001). Nature, 410, 37-40.

Clerk A and Sugden PH. (1997). Biochem. J., 325, 801-810.

Davis RJ. (2000). Cell, 103, 239-252.

Dlakic M, Grinberg AV, Leonard DA and Kerppola TK. (2001). EMBO J., 20, 828-840.

Dong C, Yang DD, Tournier C, Whitmarsh AJ, Xu J, Davis RJ and Flavell RA. (2000). Nature, 405, 91-94.

Dunn C, Wiltshire C, MacLaren A and Gillespie DA. (2002). Cell. Signal., 14, 585-593.

Eferl R and Wagner EF. (2003). Nat. Rev. Cancer, 3, 859-868.

Fuchs SY, Tappin I and Ronai Z. (2000). J. Biol. Chem., 275, $12560-12564$.

Hai T and Hartman MG. (2001). Gene, 273, 1-11.

Hall MC, Young DA, Waters JG, Rowan AD, Chantry A, Edwards DR and Clark IM. (2003). J. Biol. Chem., 278, 10304-10313.

Han Z, Boyle DL, Chang L, Bennett B, Karin M, Yang L, Manning AM and Firestein GS. (2001). J. Clin. Invest., 108, 73-81.

Hernandez-Torres J, Yunta M and Lazo PA. (2001). J. Biol. Chem., 276, 35405-35413.

Kennedy NJ and Davis RJ. (2003). Cell Cycle, 2, 199-201.

Kerppola TK and Curran T. (1993). Mol. Cell. Biol., 13, $5479-5489$.
Leppa S and Bohmann D. (1999). Oncogene, 18, 6158-6162.

Lopez-Borges S and Lazo PA. (2000). Oncogene, 19, 3656-3664.

Manning G, Whyte DB, Martinez R, Hunter $T$ and Sudarsanam S. (2002). Science, 298, 1912-1934.

Meggiato T, Calabrese F, De Cesare CM, Baliello E, Valente M and Del Favero G. (2003). Pancreas, 26, 65-70.

Neyns B, Katesuwanasing Vermeij J, Bourgain C, Vandamme B, Amfo K, Lissens W, DeSutter P, Hooghe-Peters E and DeGreve J. (1996). Oncogene, 12, 1247-1257.

Nezu J, Oku A, Jones MH and Shimane M. (1997). Genomics, 45, 327-331.

Nichols RJ and Traktman P.. (2004). J. Biol. Chem., 279, 7934-7946.

Sevilla A, Santos CR, Vega FM and Lazo PA. (2004). J. Biol. Chem., 279, 27458-27465.

Shaulian E and Karin M. (2001). Oncogene, 20, 2390-2400.

Shaulian E and Karin M. (2002). Nat. Cell. Biol., 4, E131-E136.

Shuman JD, Cheong J and Coligan JE. (1997). J. Biol. Chem., 272, 12793-12800.

Szabo E, Riffe ME, Steinberg SM, Birrer MJ and Linnoila RI. (1996). Cancer Res., 56, 305-315.

Tiniakos DG, Mellon K, Anderson JJ, Robinson MC, Neal DE and Horne CH. (1994). Br. J. Urol., 74, 757-761.

van Dam H and Castellazzi M. (2001). Oncogene, 20, 2453-2464.

Vega FM, Gonzalo P, Gaspar ML and Lazo PA. (2003). FEBS Lett., 544, 176-180.

Volm M, Koomagi R, Mattern J and Efferth T. (2002). Clin. Cancer Res., 8, 1843-1848.

Weiss L, Whitmarsh AJ, Yang DD, Rincon M, Davis RJ and Flavell RA. (2000). J. Exp. Med., 191, 139-146.

Wodrich W and Volm M. (1993). Carcinogenesis, 14, $1121-1124$

Yunta M, Oliva JL, Barcia R, Horejsi V, Angelisova P and Lazo PA. (2002). Eur. J. Biochem., 269, 1012-1021. 